Article

\title{
Imaging Noise Suppression: Fourth-Order Partial Differential Equations and Travelling Wave Solutions
}

\author{
Sameerah Jamal \\ School of Mathematics, University of the Witwatersrand, Johannesburg 2001, South Africa; \\ Sameerah.Jamal@wits.ac.za
}

Received: 16 October 2020; Accepted: 10 November 2020; Published: 12 November 2020

check for updates

\begin{abstract}
In this paper, we discuss travelling wave solutions for image smoothing based on a fourth-order partial differential equation. One of the recurring issues of digital imaging is the amount of noise. One solution to this is to minimise the total variation norm of the image, thus giving rise to non-linear equations. We investigate the variational properties of the Lagrange functionals associated with these minimisation problems.
\end{abstract}

Keywords: similarity solutions; variational norms; noise-suppressing algorithms; imaging

\section{Introduction}

Decades ago, the introduction of magnetic resonance imaging (MRI) technology revolutionised the world, primarily impacting medical diagnostics which benefited from images at the sub-millimeter scale. Since then, scanner technology has grown in leaps and bounds, but is still limited with degradation issues. A primary source of image degradation is thermal noise entering the MR data in the time domain [1]. Noise is also often described as the corrupted data or artificial imprints added to digital images by sensors [2].

To overcome such problems, the use of non-linear diffusion filters have been proposed, for further discussion and examples, we refer the reader to [3,4]. These filters involve the introduction of a small non-linear diffusion term with large gradient, $|\nabla u|$. To this end, Rudin-Osher-Fatemi [5] found that one way to achieve this is to minimise the total variation norm of the image under some given conditions. This idea inspired a number of similar models [6-8], and propelled the use of several mathematical tools for analysing non-linear diffusion filters and the algorithms to aid noise removal of digital images. In this paper, we consider the class of non-linear diffusion filters developed by [9]—comprised of a fourth-order partial differential equation (PDE) noise suppression model. We consider this noise removal model within the framework of Lie symmetries, mainly to obtain exact solutions to the model. The immense and diversified contribution of symmetries to all areas of science can be seen in, among numerous others, the works of [10-16]. An important byproduct of the Lie point symmetry method is that one may use its applications to recognise patterns in imaging $[17,18]$.

Let $u\left(x_{i}\right)$, where $i=1,2$, be a digital image and $u_{0}$ be its observation with random noise $\eta\left(x_{i}\right)$ for $\left(x_{i}\right) \in \Omega$. Noise is superimposed on the pixel intensity value by the formula

$$
u_{0}=u\left(x_{i}\right)+\eta\left(x_{i}\right)
$$

Noise recognition involves oscillating signals over small areas, so that noise removal implies filtering out high frequency signals while preserving the important features in the images [9]. Different functionals $R(u)$ measure the oscillations and a general formulation of the noise removal problem is to solve the $\min R(u)$ subject to some approximate noise level [19]. The general approach is 
to minimise the total variation norm of $\nabla u$ and use higher-order derivatives to measure oscillations. One option is to consider a Lagrange functional that is not rotational invariant [9], viz.

$$
R_{1}(u)=\int_{\Omega} L(u) d x_{i}
$$

where

$$
L(u)=\left|u_{x_{i} x_{i}}\right|,
$$

to measure oscillations in a noisy data. Another alternative would be choosing $[5,19]$

$$
R_{A}(u)=\int_{\Omega} \sqrt{u_{x_{i}}^{2}} d x_{i}
$$

or

$$
R_{B}(u)=\int_{\Omega} f(|\Delta u|) d x_{i}
$$

where $\Delta u$ denotes the Laplacian.

As these Lagrange functionals increase in complexity, as does the cost of the denoising process. For greater than 1-D and 2-D data, the number of unknowns increases and the type of functional will have a significant impact on cost. Here, we consider the constrained minimisation problem described by $R_{1}(u)$ as a $2-\mathrm{D}$ case, but a generalisation to higher dimensions is also possible.

The paper is organised as follows. In Section 2, we provide all mathematical preliminaries to tackle the underlying noise reduction problem. Section 3 lists the Lagrangians and the non-linear fourth-order PDEs associated with the function $R_{1}(u)$, with and without the addition of source terms. The variational (Noether) symmetries and conservation laws are presented in Section 4. In Section 5, some travelling wave solutions for digital images are derived. Finally, in Section 6 we conclude.

\section{Symmetry Methods: An Overview}

We first review the definitions and properties of point symmetries.

Consider a PDE with unknown function $u$ which depends on $n$ independent variables $x_{l}$, i.e., $x=\left(x_{1}, \ldots, x_{n}\right)$, respectively. Let

$$
G\left(x, u_{(k)}\right)=0
$$

be such a PDE, where $u_{(k)}$ represents the $k^{\text {th }}$ derivative of $u$ with respect to $x$. A one-parameter Lie group of transformations ( $\varepsilon$ is the group parameter) that is invariant under (3) is given by

$$
\bar{x}=\Xi(x, u ; \varepsilon) \quad \bar{u}=\Phi(x, u ; \varepsilon) .
$$

Invariance of (3) under the transformation (4) gives that any solution $u=\Theta(x)$ of (3) maps into another solution $v=\Psi(x ; \varepsilon)$ of (3). Expanding (4) around $\varepsilon=0$, we can find the infinitesimal transformations:

$$
\begin{aligned}
& \bar{x}_{l}=x_{l}+\varepsilon \xi^{l}(x, u)+\mathcal{O}\left(\varepsilon^{2}\right), \\
& \bar{u}=u+\varepsilon \eta(x, u)+\mathcal{O}\left(\varepsilon^{2}\right) .
\end{aligned}
$$

The action of the Lie group can be recovered from that of its infinitesimal generators acting on the space of independent and dependent variables. Hence, we consider the following vector field

$$
X=\xi^{l} \partial_{x_{l}}+\eta \partial_{u}
$$

The action of $\mathrm{X}$ is extended to all derivatives appearing in the equation in question through the appropriate prolongation. The infinitesimal criterion for invariance is given by

$$
\left.X\left[\operatorname{LHS} G\left(x, u_{(k)}\right)=0\right]\right|_{G\left(x, u_{(k)}\right)=0}=0
$$


A considerably detailed discussion can be found in [20].

The generalised total differentiation operator $D_{l}$ with respect to $x_{l}$ is

$$
D_{l}=\frac{\partial}{\partial x_{l}}+u_{l} \frac{\partial}{\partial u}+u_{l j} \frac{\partial}{\partial u_{j}}+\ldots
$$

and $W$ is the characteristic function

$$
W^{=} \eta-\xi^{j} u_{j}
$$

The Euler-Lagrange equations, if they exist, is given by $\delta L / \delta u=0$, where $\delta / \delta u$ is the Euler-Lagrange operator

$$
\frac{\delta}{\delta u}=\frac{\partial}{\partial u}+\sum_{s \geq 1}(-1)^{s} D_{l_{1}} \cdots D_{l_{s}} \frac{\partial}{\partial u_{l_{1} \cdots l_{s}}} .
$$

The term $L$ is referred to as a Lagrangian. If we include point dependent gauge terms $f_{1}, \ldots, f_{n}$, the Noether symmetries $X$ are given by

$$
X L+L D_{l} \xi^{l}=D_{l} f_{l}
$$

The operator in Equation (6) can be used to define the characteristic system

$$
\frac{d x_{l}}{\xi^{l}}=\frac{d u}{\eta}=\ldots
$$

whose solution provides the invariant functions

$$
W^{[r]}\left(x_{l}, u\right) .
$$

These invariants can be used in order to reduce the order of the PDE. More explanations and formulae can be found in [21].

\section{Noise Reduction PDEs}

Consider the 2D space $(n=2)$ for the functional $R_{1}(u)$, with

$$
L_{1}(u)=\sqrt{u_{x_{1} x_{1}}}+\sqrt{u_{x_{2} x_{2}}} .
$$

A fourth-order PDE arises from the Euler-Lagrange equation of (14), namely

$$
\frac{3}{8} \frac{u_{x_{i} x_{i} x_{i}}^{2}}{u_{x_{i} x_{i}}^{5 / 2}}-\frac{1}{4} \frac{u_{x_{i} x_{i} x_{i} x_{i}}}{u_{x_{i} x_{i}}^{3 / 2}}=0
$$

If a time evolving situation is required, then let the digital image $u\left(x_{i}\right)$ satisfy the following non-linear partial differential equation in $\Omega$, which is a parabolic equation with time as an evolution parameter.

We solve for $t>0,\left(x_{i}\right) \in \Omega$,

$$
u_{t}+\frac{3}{8} \frac{u_{x_{i} x_{i} x_{i}}^{2}}{u_{x_{i} x_{i}}^{5 / 2}}-\frac{1}{4} \frac{u_{x_{i} x_{i} x_{i} x_{i}}}{u_{x_{i} x_{i}}^{3 / 2}}=0 .
$$

Moreover, for finding minimum values of $R_{1}(u)$ subject to some noise level, a modification can be made to the Lagrange functional, based on the addition of source terms, namely let

$$
L_{2}\left(u, u_{x_{i} x_{i}}\right)=L_{1}(u)+\lambda_{1} u+\frac{1}{2} \lambda_{2} u^{2},
$$


where $\lambda_{1-2}$ are constants. Thus, the time evolution PDE in this instance is

$$
u_{t}+\lambda_{1}+\lambda_{2} u+\frac{3}{8} \frac{u_{x_{i} x_{i} x_{i}}^{2}}{u_{x_{i} x_{i}}^{5 / 2}}-\frac{1}{4} \frac{u_{x_{i} x_{i} x_{i} x_{i}}}{u_{x_{i} x_{i}}^{3 / 2}}=0,
$$

and the time-independent Euler-Lagrange equation of (17) is

$$
\lambda_{1}+\lambda_{2} u+\frac{3}{8} \frac{u_{x_{i} x_{i} x_{i}}^{2}}{u_{x_{i} x_{i}}^{5 / 2}}-\frac{1}{4} \frac{u_{x_{i} x_{i} x_{i} x_{i}}}{u_{x_{i} x_{i}}^{3 / 2}}=0
$$

\section{Conservation Laws}

The Noether condition (11) corresponding to the Lagrangian (14) provides an over-determined system of equations. Without presenting the details, once solved, we find the variational symmetries

$$
X^{1}=\partial_{x_{1}}, X^{2}=\partial_{x_{2}}, X^{3}=-x_{1} \partial_{x_{1}}-x_{2} \partial_{x_{2}}
$$

Each of these vector fields give a conserved form $\omega=T_{1}^{x} d x_{1}+T_{2}^{x} d x_{2}$. Specifically, the conserved forms are:

- $X^{1}$ :

$$
-\frac{1}{4} \frac{u_{x_{2}, x_{2}, x_{2}} u_{x_{1}}+2 u_{x_{1}, x_{2}} u_{x_{2}, x_{2}}}{u_{x_{2}, x_{2}}{ }^{3 / 2}} \mathrm{~d} x_{1}-\frac{1}{4} \frac{2 u_{x_{1}, x_{1}}{ }^{2}+4 \sqrt{u_{x_{2}, x_{2}}} u_{x_{1}, x_{1}}{ }^{3 / 2}-u_{x_{1}, x_{1}, x_{1}} u_{x_{1}}}{u_{x_{1}, x_{1}}{ }^{3 / 2}} \mathrm{~d} x_{2}
$$

- $X^{2}$ :

$$
\frac{1}{4} \frac{4 \sqrt{u_{x_{1}, x_{1}}} u_{x_{2}, x_{2}}{ }^{3 / 2}+2 u_{x_{2}, x_{2}}{ }^{2}-u_{x_{2}, x_{2}, x_{2}} u_{x_{2}}}{u_{x_{2}, x_{2}}{ }^{3 / 2}} \mathrm{~d} x_{1}+\frac{1}{4} \frac{u_{x_{1}, x_{1}, x_{1}} u_{x_{2}}+2 u_{x_{1}, x_{2}} u_{x_{1}, x_{1}}}{u_{x_{1}, x_{1}}{ }^{3 / 2}} \mathrm{~d} x_{2}
$$

- $X^{3}$ :

$$
\begin{aligned}
& -\frac{1}{4} \frac{4 x_{2} u_{x_{2}, x_{2}}{ }^{3 / 2} \sqrt{u_{x_{1}, x_{1}}}+2 u_{x_{2}, x_{2}}{ }^{2} x_{2}-x_{1} u_{x_{1}} u_{x_{2}, x_{2}, x_{2}}-2 u_{x_{2}, x_{2}} x_{1} u_{x_{1}, x_{2}}-x_{2} u_{x_{2}} u_{x_{2}, x_{2}, x_{2}}-2 u_{x_{2}, x_{2}} u_{x_{2}}}{u_{x_{2}, x_{2}}{ }^{3 / 2}} \mathrm{~d} x_{1} \\
& +\frac{1}{4} \frac{2 u_{x_{1}, x_{1}}{ }^{2} x_{1}+4 x_{1} u_{x_{1}, x_{1}}{ }^{3 / 2} \sqrt{u_{x_{2}, x_{2}}}-x_{1} u_{x_{1}} u_{x_{1}, x_{1}, x_{1}}-x_{2} u_{x_{2}} u_{x_{1}, x_{1}, x_{1}}-2 u_{x_{1}, x_{1}} x_{2} u_{x_{1}, x_{2}}-2 u_{x_{1}, x_{1}} u_{x_{1}}}{u_{x_{1}, x_{1}}{ }^{3 / 2}} \mathrm{~d} x_{2} .
\end{aligned}
$$

On the other hand, the Lagrangian (17) admits the variational symmetries

$$
X^{1}, X^{2}
$$

with conserved forms

- $X^{1}$ :

$$
\begin{gathered}
-\frac{1}{4} \frac{u_{x_{2}, x_{2}, x_{2}} u_{x_{1}}+2 u_{x_{1}, x_{2}} u_{x_{2}, x_{2}}}{u_{x_{2}, x_{2}}{ }^{3 / 2}} \mathrm{~d} x_{1} \\
-\frac{1}{4} \frac{2 \lambda_{2} u^{2} u_{x_{1}, x_{1}}{ }^{3 / 2}+4 \lambda_{1} u u_{x_{1}, x_{1}}{ }^{3 / 2}+4 \sqrt{u_{x_{2}, x_{2}}} u_{x_{1}, x_{1}}{ }^{3 / 2}+2 u_{x_{1}, x_{1}}{ }^{2}-u_{x_{1}, x_{1}, x_{1}} u_{x_{1}}}{u_{x_{1}, x_{1}}{ }^{3 / 2}} \mathrm{~d} x_{2}
\end{gathered}
$$

- $X^{2}$ :

$$
\frac{1}{4} \frac{2 \lambda_{2} u^{2} u_{x_{2}, x_{2}}{ }^{3 / 2}+4 \lambda_{1} u u_{x_{2}, x_{2}}{ }^{3 / 2}+4 \sqrt{u_{x_{1}, x_{1}}} u_{x_{2}, x_{2}}{ }^{3 / 2}+2 u_{x_{2}, x_{2}}{ }^{2}-u_{x_{2}, x_{2}, x_{2}} u_{x_{2}}}{u_{x_{2}, x_{2}}{ }^{3 / 2}} \mathrm{~d} x_{1}
$$




$$
+\frac{1}{4} \frac{u_{x_{1}, x_{1}, x_{1}} u_{2}+2 u_{x_{1}, x_{2}} u_{x_{1}, x_{1}}}{u_{x_{1}, x_{1}}{ }^{3 / 2}} \mathrm{~d} x_{2}
$$

\section{Exact Solutions}

Every variational symmetry is a Lie symmetry and may be used for the purposes of invariant solutions. Thus, the system (12) can be used to find invariants to reduce some of the PDEs of Section 3. A reduced equation is commonly easier to solve. In this section, we explore travelling wave solutions of the noise-suppressing PDEs.

A travelling wave solution of the Euler-Lagrange Equation (15) can be found using a reduction by $X^{1}+c X^{2}$, so that (16) transforms to

$$
(c+1)\left(-2 \frac{\mathrm{d}^{4} F(\zeta)}{\mathrm{d} \zeta^{4}} \frac{\mathrm{d}^{2} F(\zeta)}{\mathrm{d} \zeta^{2}}+3\left(\frac{\mathrm{d}^{3} F(\zeta)}{\mathrm{d} \zeta^{3}}\right)^{2}\right)=0,
$$

where $\zeta=-c x_{1}+x_{2}$ and (22) solves to give

$$
F(\zeta)=-4 c_{1} \ln \left(c_{2}+\zeta\right)+c_{3} \zeta+c_{4}
$$

A solution for the digital image is then

$$
u\left(x_{i}\right)=-4 c_{1} \ln \left(c_{2}-c x_{1}+x_{2}\right)+c_{3}\left(-c x_{1}+x_{2}\right)+c_{4},
$$

where the $c_{1-4}$ are four constants of integration.

Equation (16) admits the Lie point symmetries

$$
\begin{gathered}
X^{1}, X^{2}, X^{\infty}=K\left(x_{i}\right) \partial_{u}, X^{4}=u \partial_{u}+\frac{3}{2} t \partial_{t}, \\
X^{5}=t \partial_{t}+x_{1} \partial_{x_{1}}+x_{2} \partial_{x_{2}}, X^{6}=\partial_{t},
\end{gathered}
$$

whose Lie brackets appear in Table 1.

Table 1. Lie brackets of the symmetry algebra.

\begin{tabular}{cccccc}
\hline [,] & $\mathbf{X}^{\mathbf{1}}$ & $\mathbf{X}^{\mathbf{2}}$ & $\mathbf{X}^{\mathbf{4}}$ & $\mathbf{X}^{\mathbf{5}}$ & $\mathbf{X}^{\mathbf{6}}$ \\
\hline$X^{1}$ & 0 & 0 & 0 & $X^{1}$ & 0 \\
$X^{2}$ & 0 & 0 & 0 & $X^{2}$ & 0 \\
$X^{4}$ & 0 & 0 & 0 & 0 & $-\frac{1}{2}\left(3 X^{6}\right)$ \\
$X^{5}$ & $-X^{1}$ & $-X^{2}$ & 0 & 0 & $-X^{6}$ \\
$X^{6}$ & 0 & 0 & $\frac{3 X^{6}}{2}$ & $X^{6}$ & 0 \\
\hline
\end{tabular}

For the evolution Equation (16), we may apply the vector $X^{4}$ so that

$$
u\left(t, x_{i}\right)=h\left(x_{i}\right) t^{2 / 3} .
$$

This transformation leads to a PDE that inherits the symmetries $X^{1}$ and $X^{2}$, and admits the vector $-\frac{2}{3} h \partial_{h}+x_{i} \partial_{x_{i}}$. A travelling wave solution thereafter, again from $X^{1}+c X^{2}$ leads to the ODE

$$
c^{5}\left((c+1)\left(-6 \frac{\mathrm{d}^{4} k(\zeta)}{\mathrm{d} \zeta^{4}}\left(\frac{\mathrm{d}^{2} k(\zeta)}{\mathrm{d} p^{2}}\right)^{7 / 2}+9\left(\frac{\mathrm{d}^{3} k(\zeta)}{\mathrm{d} \zeta^{3}}\right)^{2}\left(\frac{\mathrm{d}^{2} k(\zeta)}{\mathrm{d} \zeta^{2}}\right)^{5 / 2}\right)+16\left(\frac{\mathrm{d}^{2} k(\zeta)}{\mathrm{d} \zeta^{2}}\right)^{5} k(\zeta)\right)=0
$$


with the invariant $h\left(x_{i}\right)=k(\zeta)$. Equation (25) possesses the Lie point symmetries

$$
\bar{X}^{1}=k \partial_{k}-\frac{3}{2} \zeta \partial_{\zeta}, \bar{X}^{2}=\partial_{\zeta}
$$

The commutator relation here is

$$
\left[\bar{X}^{1}, \bar{X}^{2}\right]=\frac{3}{2} \bar{X}^{2}
$$

In a similar process as above, the application of $\bar{X}^{1}$ leads to the solution

$$
u\left(t, x_{i}\right)=\left(\frac{\left((-100 c-100) \sqrt{10}\left(-c x_{1}+x_{2}\right)^{2}\right)^{2 / 3}}{100\left(-c x_{1}+x_{2}\right)^{2}}\right) t^{\frac{2}{3}}
$$

Moreover, Equation (19) also has a travelling wave solution from $X^{1}+c X^{2}$. By quadrature, Equation (19) reduces to

$$
8\left(\lambda_{2} m(\zeta)+\lambda_{1}\right)\left(\frac{\mathrm{d}^{2} m(\zeta)}{\mathrm{d} \zeta^{2}}\right)^{5 / 2}-2(c+1)\left(\frac{\mathrm{d}^{4} m(\zeta)}{\mathrm{d} \zeta^{4}} \frac{\mathrm{d}^{2} m(\zeta)}{\mathrm{d} \zeta^{2}}+3\left(\frac{\mathrm{d}^{3} m(\zeta)}{\mathrm{d} \zeta^{3}}\right)^{2}\right)
$$

which admits the Lie point symmetries

$$
\bar{X}^{2}, \bar{X}^{3}=\left(2 \lambda_{2} m+2 \lambda_{1}\right) \partial_{m}-3 \lambda_{2} \zeta \partial_{\zeta} .
$$

The non-vanishing Lie bracket is

$$
\left[\bar{X}^{2}, \bar{X}^{3}\right]=-3 \lambda_{2} \bar{X}^{3}
$$

The point symmetry $\bar{X}^{3}$ yields a solution for Equation (27), and ultimately the digital image is

$$
u\left(x_{i}\right)=-\frac{\lambda_{1}}{\lambda_{2}}+\frac{\left(-225 \sqrt{10}(c+1) \lambda_{2}^{2}\left(-c x_{1}+x_{2}\right)^{2}\right)^{2 / 3}}{225\left(-c x_{1}+x_{2}\right)^{2} \lambda_{2}^{2}} .
$$

On the other hand, the evolution equation with a source term, (18) admits the Lie point symmetries

$$
\begin{aligned}
X^{1}, X^{2}, X^{6}, X^{7} & =\left(2 \lambda_{2} u+2 \lambda_{1}\right) \partial_{u}-3 \lambda_{2}\left(x_{1} \partial_{x_{1}}+x_{2} \partial_{x_{2}}\right), X^{\infty}, \\
X^{8} & =\mathrm{e}^{-\frac{3}{2} \lambda_{2} t} \partial_{t}-\mathrm{e}^{-\frac{3}{2} \lambda_{2} t}\left(\lambda_{2} u+\lambda_{1}\right) \partial_{u},
\end{aligned}
$$

with Lie brackets in Table 2.

Table 2. Lie brackets of the symmetry algebra.

\begin{tabular}{cccccc}
\hline [,] & $\mathbf{X}^{\mathbf{1}}$ & $\mathbf{X}^{\mathbf{2}}$ & $\mathbf{X}^{\mathbf{6}}$ & $\mathbf{X}^{7}$ & $\mathbf{X}^{\mathbf{8}}$ \\
\hline$X^{1}$ & 0 & 0 & 0 & $-3 \lambda_{2} X^{1}$ & 0 \\
$X^{2}$ & 0 & 0 & 0 & $-3 \lambda_{2} X^{2}$ & 0 \\
$X^{6}$ & 0 & 0 & 0 & 0 & $-\frac{1}{2}\left(3 \lambda_{2} X^{8}\right)$ \\
$X^{7}$ & $3 \lambda_{2} X^{1}$ & $3 \lambda_{2} X^{2}$ & 0 & 0 & 0 \\
$X^{8}$ & 0 & 0 & $\frac{3 \lambda_{2} X^{8}}{2}$ & 0 & 0 \\
\hline
\end{tabular}


Hence, we may obtain an exact solution for (18) with the use of the vector field $X^{8}$ - that is, Equation (18) transforms to the PDE

$$
3\left(\frac{\partial^{2} w\left(q_{i}\right)}{\partial q_{r}{ }^{2}}\right)^{5 / 2}\left(\frac{\partial^{3} w\left(q_{i}\right)}{\partial q_{i}{ }^{3}}\right)^{2}-2\left(\frac{\partial^{2} w\left(q_{i}\right)}{\partial q_{1}{ }^{2}}\right)^{5 / 2} \frac{\partial^{4} w\left(q_{i}\right)}{\partial q_{2}{ }^{4}} \frac{\partial^{2} w\left(q_{i}\right)}{\partial q_{2}{ }^{2}}=0, \quad r=1,2 i \neq r
$$

This PDE has the Lie point symmetries

$$
\bar{X}^{4}=q_{1} \partial_{q_{1}}+q_{2} \partial_{q_{2}}, \bar{X}^{5}=\partial_{q_{1}}, \bar{X}^{6}=\partial_{q_{2}}, \bar{X}^{\infty}=J\left(q_{i}\right) \partial_{w}
$$

with non-zero commutators

$$
\left[\bar{X}^{4}, \bar{X}^{5}\right]=\bar{X}^{4}, \quad\left[\bar{X}^{5}, \bar{X}^{6}\right]=\bar{X}^{5}
$$

Therefore, a travelling wave solution is obtainable from $\bar{X}^{5}+c \bar{X}^{6}$, where $q_{i} \equiv x_{i}$ and

$$
\begin{aligned}
w\left(q_{i}\right)= & \frac{1}{6} \frac{(3 c+1)^{2}\left(c_{2}-c q_{1}+q_{2}\right)^{2} 4^{(3 c+1)^{-1}}}{c(-1+3 c)} c_{1}{ }^{3 \frac{c}{3 c+1}}\left(-\frac{1}{(3 c+1)\left(c_{2}+\left(-c q_{1}+q_{2}\right)\right)}\right)^{2(3 c+1)^{-1}} \\
& +c_{3}\left(-c q_{1}+q_{2}\right)+c_{4} .
\end{aligned}
$$

That leaves us with the digital image solution of

$$
u\left(t, x_{i}\right)=\frac{\mathrm{e}^{-\lambda_{2} t} w\left(q_{i}\right) \lambda_{2}-\lambda_{1}}{\lambda_{2}}
$$

The corresponding invariant transformations of every symmetry generator is provided in Table 3.

Table 3. Invariants of the symmetry algebras. PDE: partial differential equation; ODE: ordinary differential equation.

\begin{tabular}{ccc}
\hline PDEs/ODEs Dependency & Point Symmetry & Invariants \\
\hline Time-independent & $X^{1}$ & $x_{2}, u\left(x_{i}\right)$ \\
& $X^{2}$ & $x_{1}, u\left(x_{i}\right)$ \\
& $X^{3}$ & $\frac{x_{2}}{x_{1}}, u\left(x_{i}\right)$ \\
& $\bar{X}^{1}$ & $k(\zeta) \zeta^{2 / 3}$ \\
& $\bar{X}^{2}$ & $k(\zeta)$ or $m(\zeta)$ \\
& $\bar{X}^{3}$ & $\frac{\zeta^{2 / 3}\left(\lambda_{2} m(\zeta)+\lambda_{1}\right)}{\lambda_{2}}$ \\
& $\bar{X}^{4}$ & $q_{2}, w\left(q_{i}\right)$ \\
& $\bar{X}^{5}$ & $q_{1}, w\left(q_{i}\right)$ \\
& $\bar{X}^{6}$ & $\frac{q_{2}}{q_{1}}, w\left(q_{i}\right)$ \\
\hline Time-dependent & $X^{1}$ & $x_{2}, t, u\left(t, x_{i}\right)$ \\
& $X^{2}$ & $x_{1}, t, u\left(t, x_{i}\right)$ \\
& $X^{4}$ & $x_{1}, x_{2}, u\left(t, x_{i}\right) t^{-2 / 3}$ \\
$X^{5}$ & $\frac{x_{2}}{x_{1}}, \frac{t}{x_{1}}, u\left(t, x_{i}\right)$ \\
& $X^{6}$ & $x_{1}, x_{2}, u\left(t, x_{i}\right)$ \\
& $X^{7}$ & $t, \frac{x_{2}}{x_{1}}, \frac{x_{1}^{2 / 3}\left(\lambda_{2} u\left(t, x_{i}\right)+\lambda_{1}\right)}{\lambda_{2}}$ \\
& $X^{8}$ & $x_{1}, x_{2}, \frac{e^{\lambda_{2}}\left(\lambda_{2} u\left(t, x_{i}\right)+\lambda_{1}\right)}{\lambda_{2}}$ \\
\hline
\end{tabular}

\section{Conclusions}

The focus of this study was to explore travelling wave solutions of PDEs found in image processing. As these models are highly non-linear, an advantageous approach of dealing with them is to consider their invariance properties. This entails a Lie or Noether symmetry classification, which was applied 
to a static and non-static fourth-order PDE. Additionally, we looked at several solutions in the case of the PDEs having a source term. Our application of Lie's theory provided the four digital image solutions, whereas the use of Noether's theorem enabled us to obtain conservation laws of the Lagrange functionals. Both the evolution PDEs admitted a five-dimensional Lie algebra (excluding the $X^{\infty}$ vector). The invariants of the symmetry classification are given so that other solutions, not presented here, can be calculated.

To perceive our solutions compared to existing noise suppression, consider Figure 1. The first graph is simply a noisy data set and (b) is the Gaussian function $G=\frac{1}{\sqrt{4 \pi t}} e^{-\frac{x^{2}}{4 t}}$ which is a solution of the heat equation $u_{t}=\Delta u$. The heat equation is the benchmark for noise smoothing, eliminating noise but leaving blurry edges at times. In (b), as expected we observe a smoothing of the image and interestingly, (c) also has this feature. We conclude that these solutions are effective in noise smoothing.

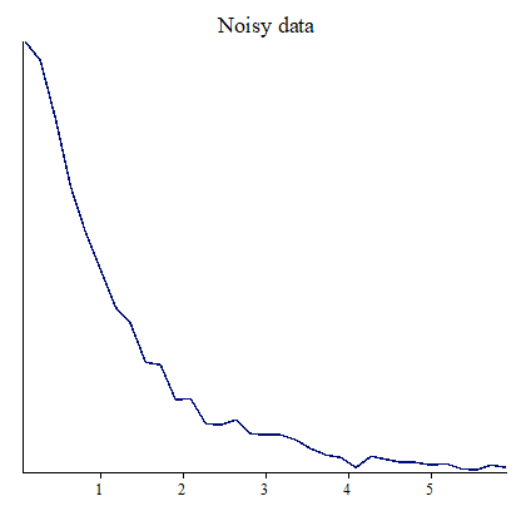

(a)

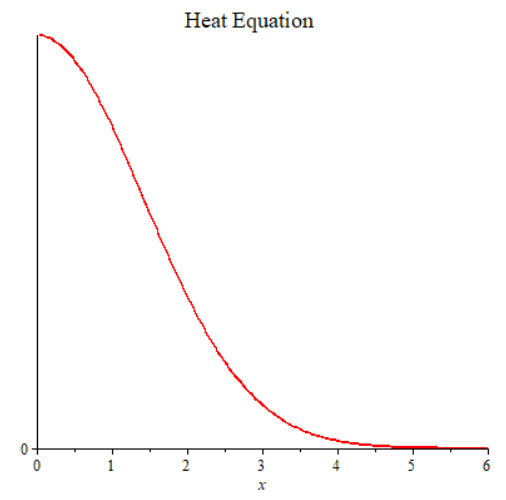

(b)

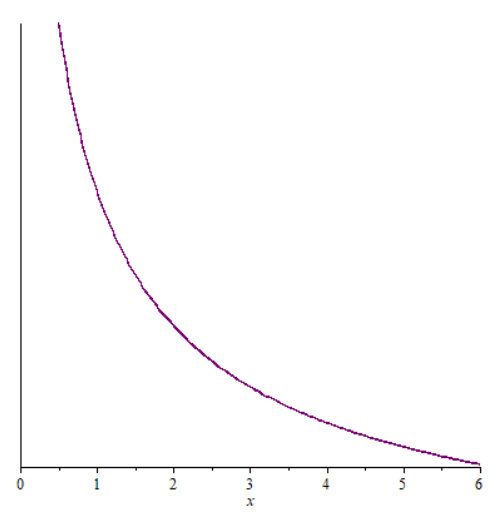

(c)

Figure 1. Smoothing and edge enhancement in 1D. We select the parameter values $\lambda_{1}=1$, $\lambda_{2}=-1, c=-4, t=x_{2}=1$ : (a) a noisy data set; (b) Gaussian solution of the heat equation; (c) the solution (26), note that (28) is similar.

Some limitations of this study include: (a) the model and our results are only relevant to post-processing noise reduction, (b) this particular noise suppression PDE, like most other denoising methods, does not incorporate signal-dependent noise characteristics (or so-called noise-statistic subtleties) in the model, and (c) to further evaluate the performance of the PDE model, one must consider the signal-to-noise ratio.

The results of this study can be further tested on a range of medical MRIs and synthesised images so that improvements can be made by considering boundary preservation, computational costs and applicability to various image types. 
In medical diagnostics in particular, noise reduction is an expensive process. It is therefore paramount to have a denoising model with efficient computing time, which suppresses noise and produces good edges. In 2D, digital images have very similar spatial resolution along the $x$ and $y$ dimensions. However, in medical MRI applications, volumetric 3D images and also time series of volume images in $4 \mathrm{D}$ are also important. Fortunately, the method used to solve above can be extended to include higher dimensional data. However, as noise functionals get more complex, the computing time grows as well. In 1D and 2D data sets, this is not a big concern, but as the dimensions and unknown parameters increase, the functional will have a dramatic effect on computing time. Regarding the computational efficiency, the fourth-order scheme is slightly slower than the second-order schemes that exist in the literature. This is due to the complexity of the PDE in terms of the presence of mixed derivatives. Thus, our fourth-order model would incur higher computational costs. However, we emphasise that our reduced equations and solutions diminish some of these costs of processing data.

The symmetry and conservation law analysis of the variational noise functionals provide a rigorous mathematical tool to introduce solutions to the digital image. In conclusion, our graphical analysis demonstrates that our results are robust and exhibit good noise suppression, and more importantly, preserve significant structural details in images. In future work, we will explore several other noise associated variational functionals as well as higher spatial dimensions.

Funding: This work has received financial support from the National Research Foundation of South Africa (99279). Conflicts of Interest: The author declares no conflict of interest.

\section{References}

1. Haacke, E.M.; Brown, R.W.; Thompson, M.R.; Venkatesan, R. Magnetic Resonance Imaging: Physical Principles and Sequence Design; Wiley: New York, NY, USA, 1999.

2. Marr, D.; Hildreth, E. Theory of Edge Detection. Proc. R. Soc. Lond. Ser. B 1980, 207, 187-217.

3. Perona, A.; Malik, J. Scale-space and edge detection using anisotropic diffusion. IEEE Trans. Pattern Anal. Mach. Intell. 1990, 12, 629-639. [CrossRef]

4. Gerig, G.; Kubler, O.; Kikinis, R.; Jolesz, F. Nonlinear anisotropic filtering of MRI data. IEEE Trans. Med. Imaging 1992, 11, 221-232. [CrossRef] [PubMed]

5. Rudin, L.I.; Osher, S.; Fatemi, E. Nonlinear total variation based noise removal algorithms. Physica D 1992, 60, 259-268. [CrossRef]

6. Chambolle, A.; Lions, P.-L. Image recovery via total variation minimization and related problems. Numer. Math. 1997, 76, 167-188. [CrossRef]

7. Alvarez, L.; Lions, P.-L.; Morel, J.-M. Image selective smoothing and edge detection by nonlinear diffusion: II. SIAM J. Numer. Anal. 1992, 29, 845-866. [CrossRef]

8. Osher, S.; Solé, A.; Vese, L. Image decomposition and restoration using total variation minimization and the $\mathrm{H}^{-1}$-norm. Multiscale Model. Simul. 2003, 1, 349-370. [CrossRef]

9. Lysaker, M.; Lundervold, A.; Tai, X.-C. Noise Removal Using Fourth-Order Partial Differential Equation with Applications to Medical Magnetic Resonance Images in Space and Time. IEEE Trans. Image Process. 2003, 12, 1579-1590. [CrossRef] [PubMed]

10. Dimakis, N.; Giacomini, A.; Jamal, S.; Leon, G.; Paliathanasis, A. Noether symmetries and stability of ideal gas solutions in Galileon cosmology. Phys. Rev. D 2017, 95, 064031. [CrossRef]

11. Leach, P.G.L.; Bouquet, S. Symmetries and integrating factors. J. Nonl. Math. Phys. 2002, 9, 73-91. [CrossRef]

12. Jamal, S. A study of the approximate singular Lagrangian-conditional Noether symmetries and first integrals. Int. J. Geom. Methods Mod. Phys. 2019, 16, 1950033. [CrossRef]

13. Shabbir, G.; Ramzan, M.; Hussain, F.; Jamal, S. Classification of static spherically symmetric space-times in $\mathrm{f}(\mathrm{R})$ theory of gravity according to their conformal vector fields. Int. J. Geom. Methods Mod. Phys. 2018, 15, 1850193. [CrossRef]

14. Gandarias, M.L.; Bruzón, M.S. Conservation laws for a Boussinesq equation. Appl. Math. Nonlinear Sci. 2017, 2, 465-472. [CrossRef] 
15. Jamal, S. A group theoretical application of $\mathrm{SO}(4,1)$ in the de Sitter universe. Gen. Relativ. Gravit. 2017, 49,1-14. [CrossRef]

16. Al Qurashi, B.; Kara, A.H.; Akca, H. mage Processing and 'Noise Removal Algorithms' The Pdes and Their Invariance Properties \& Conservation Laws. Acta Appl. Math. 2018, 153, 163.

17. Bigün, J. Pattern recognition in images by symmetries and coordinate transformations. Comput. Vis. Image Underst. 1997, 68, 290-307. [CrossRef]

18. Bigün, J. Local Symmetry Features in Image Processing, Linköping Studies in Science and Technology Dissertations No. 179, Linköping. 1989. Available online: http://ieeexplore.ieee.org (accessed on 12 November 2020).

19. You, Y.-L.; Kaveh, M. Fourth-order partial differential equation for noise removal. IEEE Trans. Image Process. 2000, 9, 1723-1730. [CrossRef] [PubMed]

20. Olver, P.J. Application of Lie Groups to Differential Equations; Springer: New York, NY, USA, 1993.

21. Stephani, H. Differential Equations: Their Solutions Using Symmetry; Cambridge University Press: Cambridge, UK, 1989.

Publisher's Note: MDPI stays neutral with regard to jurisdictional claims in published maps and institutional affiliations.

(C) 2020 by the author. Licensee MDPI, Basel, Switzerland. This article is an open access article distributed under the terms and conditions of the Creative Commons Attribution (CC BY) license (http:/ / creativecommons.org/licenses/by/4.0/). 